\title{
SELEÇÃO DE MODELOS DE REGRESSÃO PARA ESTIMAR A ÁREA FOLIAR DE MACIEIRAS 'ROYAL GALA' E 'FUJI SUPREMA' SOB TELA ANTIGRANIZO E EM CÉU ABERTO ${ }^{1}$
}

\author{
LEOSANE CRISTINA BOSCO ${ }^{2}$, HOMERO BERGAMASCHI ${ }^{3}$, LOANA SILVEIRA CARDOSO ${ }^{4}$, \\ VIVIANE AIRES DE PAULA ${ }^{5}$, BRUNO CASAMALI ${ }^{6}$
}

RESUMO - O objetivo deste trabalho foi ajustar e testar modelos matemáticos simples, precisos e acurados para estimar a área foliar de macieiras 'Royal Gala' e 'Fuji Suprema' cultivadas sob tela antigranizo e em céu aberto. O experimento foi conduzido em pomar comercial no município de Vacaria-RS, nos ciclos vegetativos de 2008/2009 e 2009/2010. Equações de regressão linear e quadrática foram ajustadas para estimativa da área foliar em função das dimensões das folhas. A seleção dos melhores modelos baseou-se no quadrado médio do erro, no coeficiente de determinação, no erro-padrão da estimativa e na significância dos coeficientes das equações. A área foliar estimada pelos melhores modelos foi comparada com dados obtidos de medição por planímetro e estimados por fotografias digitais. A prova dos modelos baseou-se na significância do coeficiente angular, na linha 1:1 e na raiz quadrada do quadrado médio do erro. Modelos linear e quadrático que consideram o comprimento e a largura das folhas estimam com acurácia e precisão a área foliar de macieiras 'Royal Gala' e 'Fuji Suprema', tanto sob tela antigranizo quanto em céu aberto. A área foliar de macieira estimada por modelos de regressão que utilizam comprimento e largura de folhas é similar à obtida por planímetro ou fotografia digital.

Termos para indexação: Malus domestica, modelos de regressão, folhas, comprimento, largura.

\section{SELECTION OF REGRESSION MODELS FOR ESTIMATING LEAF AREA OF 'ROYAL GALA' AND 'FUJI SUPREMA' APPLE TREES UNDER HAIL NET AND IN OPEN SKY}

\begin{abstract}
The objective of this study was to adjust and to test simple, precise, and accurate mathematical models for estimating the leaf area of 'Royal Gala' and 'Fuji Suprema' apple trees grown under coverage of black hail net and in open sky. The experiment was carried out in a commercial apple orchard, in Vacaria, Brazil, during the vegetative cycles of 2008/2009 and 2009/2010. Linear and quadratic regression equations were adjusted for estimating the leaf area in function of leaf dimensions. The selection for best models was based on mean square error, determination coefficient, standard error of estimative, and significance of equation coefficients. The leaf area estimated by the best models was compared with data obtained by electronic planimeter and estimated by digital photography. The prove of models was based on the significance of the angular coefficient, on the 1:1 line, and on the root mean square error. Linear and quadratic models that consider the length and width of leaves estimated accurately and precisely the leaf area of 'Royal Gala' and 'Fuji Suprema' apple trees for both cropping conditions, open sky or under hail net. The apple trees leaf area estimated by regression models using leaf length and width is similar to those obtained by planimetry or digital photography.
\end{abstract}

Index terms: Malus domestica, regression models, leaf, length, width.

\footnotetext{
'(Trabalho 217-11). Recebido: 29-08-2011. Aceito para publicação em: 09-05-2012.

2Eng. Agrônoma, Doutoranda do PPG Fitotecnia, Faculdade de Agronomia, UFRGS, Av. Bento Gonçalves, 7712, CEP: 91540-000, Porto Alegre-RS. e-mail: leosaneb@yahoo.com.br - Bolsista CNPq

${ }^{3}$ Eng. Agrônomo, Prof. Doutor, Dep. Plantas Forrageiras e Agrometeorologia, Faculdade de Agronomia, UFRGS, Av. Bento Gonçalves, 7712, CEP: 91540-000, Porto Alegre-RS. e-mail: homerobe@ufrgs.br - Bolsista CNPq

${ }^{4}$ Eng. Agrônoma, Doutoranda do PPG Fitotecnia, Faculdade de Agronomia, UFRGS, Av. Bento Gonçalves, 7712, CEP: 91540-000, Porto Alegre-RS. e-mail: loanacar@yahoo.com.br - Bolsista CNPq

${ }^{5}$ Eng. Agrônoma, Doutoranda do PPG Fitotecnia, Faculdade de Agronomia, UFRGS, Av. Bento Gonçalves, 7712, CEP: 91540-000, Porto Alegre-RS. e-mail: vivianeadp@yahoo.com.br - Bolsista CNPq

${ }^{6}$ Acadêmico de Agronomia, UFRGS, Av. Bento Gonçalves, 7712, CEP: 91540-000, Porto Alegre-RS. e-mail: bruno.casa@terra.com.br Bolsista Iniciação Científica CNPq.
} 


\section{INTRODUÇÃO}

Medidas simples e acuradas de área foliar são de interesse para pesquisas que envolvem as interações entre o crescimento das plantas e seu ambiente. Devido à sua relação direta com a fotossíntese, a área foliar é determinante em processos relacionados ao metabolismo, acúmulo de biomassa, fenologia e rendimento dos cultivos (DEMIRSOY, 2009). Sendo assim, ela é importante em estudos que caracterizam os ecossistemas e processos relacionados a temas como ecofisiologia, nutrição, competição intra e interespecífica de plantas, epidemiologia, melhoramento genético e manejo dos cultivos (PALMER, 1987).

Com a evolução da pomicultura no Brasil, surgiu a necessidade de aumentar a produtividade dos pomares. A utilização de porta-enxertos menos vigorosos e a condução das plantas em líder central permitiram aumentar a densidade de plantas e, em consequência, a produtividade. Por outro lado, a área foliar total foi reduzida, promovendo maior penetração de radiação solar, maior ventilação e modificações de características morfológicas e fisiológicas do dossel (BALAN, 2005). Isto despertou novos interesses por pesquisas básicas relacionadas ao crescimento e ao desenvolvimento das macieiras. A relação entre área foliar e produção de frutos também é importante, pois ela influencia o crescimento e a qualidade dos frutos.

Em plantas caducifólias, como a macieira, a cada ano há emissão de um novo conjunto de folhas, que podem variar em número e tamanho, influenciando processos fisiológicos importantes. Segundo Palmer (1987), existe variabilidade na área foliar devido a diferenças de cultivares, idade das plantas, condições relacionadas a safras, locais e espaçamentos entre plantas. Outro fator que pode influenciar a área foliar é o cultivo em ambientes protegidos. Estudos realizados no Rio Grande do Sul demonstraram que videiras cultivadas sob cobertura plástica apresentam folhas maiores e com maior comprimento de entrenós (CARDOSO, 2007; COMIRAN, 2009; CARDOSO et al., 2010). Na Austrália, plantas de macieira cultivadas sob tela antigranizo também apresentam folhas maiores, além de um maior número e comprimento de brotos (MIDDLETON; McWATERS, 2002).

A determinação de área foliar pode ser feita pelo uso de instrumentos de medição ou de modelos de regressão. Métodos destrutivos para medição da área foliar incluem o uso de scanners, câmeras fotográficas ou planímetros. Estes métodos, porém, não são adequados a medições de acompanhamento do crescimento das plantas (LU et al., 2004), além de danificarem as folhas e tornarem as avaliações trabalhosas e demoradas. Recentemente, instrumentos baseados em laser foram desenvolvidos para medir a área foliar em dosséis de culturas e florestas (PINTO JÚNIOR et al., 2010; FLADUNG; RITTER, 1991). No entanto, esses instrumentos são caros e de uso complexo (O'NEAL et al., 2002). Métodos de medição não destrutivos podem ser mais eficientes, pois, além de não comprometerem a avaliação de outros parâmetros dependentes da área foliar, reduzem a variabilidade associada a procedimentos de amostragem (LIMA E SILVA et al., 2004).

O emprego de modelos de regressão para estimar a área foliar é uma alternativa não destrutiva simples, rápida, acurada, confiável e barata (PINTO et al., 2004). Os procedimentos usuais deste método envolvem medidas de comprimento, largura e área foliar de amostras de folhas, a fim de ajustar equações de regressão e escolher aquelas que apresentam melhor precisão estatística para estimar a área de amostras subsequentes (WIERSMA; BAILEY, 1975). Para obter medidas acuradas de área foliar em macieira, o método mais simples baseia-se na contagem do número de folhas de algumas plantas do pomar e na medição das dimensões de uma amostra de folhas, sem a necessidade de retirá-las das plantas. Essas dimensões são utilizadas como variáveis explicativas em modelos matemáticos específicos, para estimar a área foliar da macieira.

Modelos matemáticos baseados em medidas lineares do limbo foliar (comprimento e largura) têm sido ajustados para estimar a área de folhas em plantas daninhas (BIANCO et al., 2004), cultivos agrícolas (MALDANER et al., 2009; ADAMI et al., 2008; DE JESUS et al., 2001; LAKITAN, 1989; FRANCIS et al., 1969; TAVARES-JÚNIOR et al., 2002), espécies hortículas (PEKSEN, 2007; QUEIROGA et al., 2003; UZUN; ÇELIK, 1999; BEYHAN et al., 2008), plantas ornamentais (PINTO et al., 2004; SILVA et al., 2008) e pastagens (FAGUNDES et al., 2001). Em espécies arbóreas e frutíferas, foram ajustados modelos para determinar a área foliar de videira (PEDRO JÚNIOR et al., 1986; GONÇALVES et al., 2002; CARDOSO, 2007; COMIRAM, 2009), bananeira (POTDAR; PAWAR, 1991), frutíferas silvestres (MIELKE et al., 1995), cajueiro (LIMA E SILVA et al., 2000), mamoeiro (CAMPOSTRINI; YAMANISHI., 2001), pessegueiro (DEMIRSOY et al., 2004), fruta-do-conde (LIMA;SILVA et al., 2004), lima-ácida (COELHO FILHO et al., 2005), castanheira (SERDAR; DEMIRSOY, 2006), aveleira (CRISTOFORI et al., 2007) e nogueira (TORRI et al., 2009). Palmer (1987) e Demirsoy (2009) estudaram modelos de área foliar para macieira levando em 
consideração a forma e o tamanho da copa das plantas e não as dimensões lineares do limbo foliar. Em Santa Catarina, Leonetti et al. (2008) ajustaram equações lineares para estimar a área foliar em quatro cultivares de macieira, mas esses modelos não tiveram testes de validação com dados estimados por outros métodos, para verificar sua acurácia e precisão.

Devido à importância da área foliar como parâmetro de crescimento da macieira, o objetivo deste trabalho foi ajustar e testar modelos matemáticos simples, precisos e acurados para estimar a área foliar de macieira 'Royal Gala' e 'Fuji Suprema' cultivadas sob tela antigranizo e em céu aberto.

\section{MATERIAL E MÉTODOS}

O estudo foi conduzido em pomar comercial de macieira localizado no município de Vacaria-RS, a $930 \mathrm{~m}$ de altitude, $28^{\circ} 22^{\prime} 52,1^{\prime \prime} \mathrm{S}$ e $50^{\circ} 50^{\prime} 46,3^{\prime \prime} \mathrm{W}$, durante os ciclos produtivos de 2008/2009 e 2009/2010. As cultivares avaliadas foram 'Royal Gala' e 'Fuji Suprema' sobre o porta-enxerto M9, ambas com dez anos de idade, conduzidas em líder central com apoio. O sistema de cultivo foi de alta densidade, com espaçamento de 1,0 m entre plantas e de 3,5 m entre linhas. Para cada cultivar, foram avaliados dois ambientes de cultivo, sendo um em céu aberto e outro sob tela antigranizo. Para a cobertura do pomar, foi utilizada tela de cor preta, com malha de $4 \times 7 \mathrm{~mm}$ sobre uma estrutura fixa desde o ano de implantação do pomar.

No ciclo produtivo de 2008/2009, foram coletadas folhas totalmente expandidas e sadias, em ambos os ambientes e em diferentes épocas (outubro, dezembro e janeiro). As folhas foram selecionadas ao acaso, em diferentes posições da planta. Para o ajuste do modelo de estimativa da área foliar, foram coletadas cerca de 1.000 folhas, sendo 345 folhas de 'Royal Gala' e 150 de 'Fuji Suprema', em cada ambiente de cultivo.

Imediatamente após a coleta, as folhas foram acondicionadas em sacos de plástico e transportadas ao laboratório, para medição do comprimento $(\mathrm{C})$, largura (L) e área foliar (AF). O comprimento da folha foi medido da ponta da lâmina até a inserção do pecíolo, ao longo da nervura central. A largura foi medida entre os lóbulos maiores da lâmina, perpendicularmente à nervura central. As medições de $\mathrm{C} e$ $\mathrm{L}$ foram feitas com régua graduada em milímetros e a área foliar foi medida usando-se um planímetro eletrônico da marca LI-COR, modelo LI 3000.

Para caracterizar o formato das folhas, dos diferentes ambientes e cultivares, determinou-se o índice de forma (IF) pela relação C/L. Este índice caracteriza o formato foliar, pelos seguintes critérios: folhas com valores de IF inferiores a 1,0 são mais largas que longas; folhas com IF superiores a 1,0 são proporcionalmente mais alongadas e estreitas. Determinou-se também a área foliar média com dados de medição de AF (por planímetro).

A fim de verificar diferenças entre os ambientes sob tela antigranizo e em céu aberto, as médias desses tratamentos foram comparadas pelo teste $\mathrm{F}$, ao nível de $5 \%$ de probabilidade, considerando as variáveis comprimento, largura, índice de forma e área foliar média de folhas de macieiras 'Royal Gala' e 'Fuji Suprema', coletadas em 2008/2009. A análise estatística foi realizada separadamente para cada cultivar, considerando-se uma fonte de variação, o ambiente. Para 'Royal Gala' e 'Fuji Suprema' foram colhidas ao acaso 115 e 50 folhas, respectivamente, tanto sob tela antigranizo quanto em céu aberto, em cada uma das três épocas de coleta. As médias das variáveis obtidas em cada época foram consideradas as repetições.

Antes do ajuste dos modelos, os dados de comprimento, largura e área foliar foram submetidos aos testes de Bartlett, Kolmogorov-Smirnov e Durbin-Watson para, respectivamente, verificar a homogeneidade de variância, a distribuição normal dos resíduos e a independência dos dados $(\mathrm{SCH}-$ NEIDER, 1998). Se o conjunto de dados apresentar características de homogeneidade de variância, distribuição normal e independência, estes serão utilizados sem nenhuma transformação.

Para o ajuste dos modelos, procederam-se a análises de regressão linear e quadrática, com passagem forçada da função pela origem, considerada como a mais apropriada para estimar a área foliar (LAKITAN, 1989; LIMA E SILVA et al., 2000; LIMA E SILVA et al., 2004). Este procedimento apresenta bases geométricas aceitáveis, não altera expressivamente a soma de quadrado dos resíduos e torna o modelo mais prático para sua utilização (BIANCO et al., 2004). A variável dependente (área foliar - AF) foi relacionada com diferentes variáveis independentes (comprimento - C, largura - L e produto $\mathrm{C} \mathrm{x} \mathrm{L)}$.

Foram efetuados cálculos para detectar a colinearidade entre as variáveis $\mathrm{C}$ e $\mathrm{L}$. Para isto, foram calculados o fator de inflação da variância $\left[F I V=1 /\left(1-r^{2}\right)\right]$ e o valor de tolerância $(T=1 / F I V)$, sendo $r$ o coeficiente de correlação. Se FIV for maior que 10 ou se $\mathrm{T}$ for menor que 0,10 , a colinearidade influencia os parâmetros de estimativa do modelo e, portanto, o modelo deve ser excluído (CRISTOFORI et al., 2007).

A decisão para a escolha do melhor modelo de estimativa da área foliar foi baseada no quadrado 
médio do erro (QME), no coeficiente de determinação $\left(\mathrm{R}^{2}\right)$, no erro-padrão da estimativa $(\mathrm{EP})$ e na significância do coeficiente linear $(b)$ em modelos lineares e no coeficiente quadrático $(c)$ em modelos quadráticos. O melhor modelo foi aquele com maior $\mathrm{R}^{2}$, menores QME e EP, e com valor dos coeficientes $b$ e $c$ mais próximos de 1,0 . Esta avaliação estatística foi realizada para cada cultivar, em cada ambiente de cultivo, para todo o conjunto de dados. Os melhores modelos foram organizados em ordem de classificação, sendo que o melhor ajuste para cada modelo foi escolhido para a realização dos testes de validação e desempenho.

A validação e o desempenho dos modelos selecionados foi realizada com dados de comprimento, largura e área foliar obtidos de 650 folhas de cada cultivar, no ciclo de 2009/2010. Nesse ciclo, as medições foliares foram realizadas conforme a metodologia adotada no ciclo de 2008/2009. A área de cada folha, individualmente, que foi estimada pelos melhores modelos, foi comparada com a área foliar real obtida por medições em planímetro, estimada por meio de fotografias digitais e pelo modelo de Leonetti et al. (2008). Para a determinação da área foliar por meio de fotografias, foi utilizada uma câmera fotográfica digital com resolução de seis megapixels, sem uso de flash, e instalada sobre um suporte fixo à distância de $25 \mathrm{~cm}$ da folha. Após a aquisição das fotos, utilizou-se o programa QUANT 1.0.1 (VALE et al., 2003) para determinar a área correspondente a cada folha. As folhas foram enumeradas na face abaxial, para serem utilizadas nas determinações da área foliar, a partir das medidas de $\mathrm{C}$ e $\mathrm{L}$, obtidas por régua, e da área determinada por planímetro e por fotografias. O modelo de Leonetti et al. (2008) foi gerado com dados da cultivar 'Royal Gala', e consiste na seguinte fórmula: $\mathrm{AF}=$ $0,7241 * \mathrm{CL}\left(\mathrm{R}^{2}=0,98\right)$.

A validação do modelo baseou-se na significância dos coeficientes linear $(b)$ e quadrático (c), e sua correspondência, à linha 1:1. Além disso, calculou-se o valor da raiz quadrada do quadrado médio do erro (RQME), pela fórmula: $\mathrm{RQME}=$ $\left(\Sigma\left(\mathrm{S}_{\mathrm{i}}-\mathrm{O}_{\mathrm{i}}\right)^{2} / \mathrm{N}\right)^{0,5}$, em que, $\mathrm{S}_{\mathrm{i}}$ é o iésimo valor estimado de área foliar; $\mathrm{O}_{\mathrm{i}}$ é o iésimo valor observado de área foliar, e N é o número de observações. Assim, quanto menor for RQME, melhor é o ajuste do modelo (JANSSEN; HEUBERGER, 1995).

$\mathrm{O}$ confronto entre valores de área foliar medidos por instrumento de medida (planímetro) e estimados por análises de regressão linear permitiu avaliar o desempenho de cada modelo ajustado. Este desempenho foi testado por meio das seguintes estatísticas: coeficiente de correlação (r), que indica a precisão; índice de Willmott (d), que indica exatidão ou concordância da estimativa das variáveis (WILLMOTT, 1981); e o índice de confiança (c), que indica confiança ou desempenho do modelo (CAMARGO; SENTELHAS, 1997).

\section{RESULTADOS E DISCUSSÃO}

Os dados de comprimento (C), largura (L) e área foliar (AF) apresentaram homogeneidade de variância, distribuição normal e independência dos dados, conforme os resultados obtidos pelos testes de Bartlett, Kolmogorov-Smirnov e Durbin-Watson, não sendo necessária transformação dos dados.

Entre ambientes de cultivo, não se observaram diferenças significativas para as variáveis comprimento, largura, índice de forma e área foliar média de folhas de macieiras, tanto para as médias da cultivar 'Royal Gala' quanto para as médias de 'Fuji Suprema' (Tabela 1). Esta é uma evidência de que não há necessidade de diferentes modelos para estimar a área foliar de macieiras cultivadas em diferentes ambientes, sob tela antigranizo e em céu aberto.

Com base no grau de colinearidade entre $\mathrm{C}$ e L, a avaliação dos dados mostrou que o fator de inflação da variância (FIV) foi superior a 10,0 e que o valor de tolerância (T) foi inferior a 0,10 para a cultivar 'Fuji Suprema', tanto em céu aberto como sob tela antigranizo (Tabela 2). Portanto, existe colinearidade entre as variáveis C e L da cultivar 'Fuji Suprema'. Sendo assim, para esta cultivar, podem ser ajustados modelos com apenas uma dimensão foliar, $\mathrm{C}$ ou L.

Os 36 modelos gerados apresentaram significância estatística, com $\mathrm{R}^{2}$ igual ou superior a 0,70 , EP igual ou inferior a 5,7 e QME igual ou inferior a 32,45 (Tabela 3). Sendo assim, modelos classificados a partir da quinta colocação podem ser utilizados para estimar a área foliar em estudos que toleram menor precisão de resultados. Modelos que utilizaram $\mathrm{C}$ ou L apresentaram menor significância em comparação a modelos com as duas dimensões. Nos modelos lineares com apenas uma dimensão foliar, obteve-se menor significância do coeficiente $b$, com $\mathrm{R}^{2}$ entre 0,70 e 0,77 , e EP igual ou superior a 4,23 (Tabela 3 ). Nos modelos quadráticos com apenas uma dimensão foliar, os coeficientes foram altamente significativos, com $\mathrm{R}^{2}$ igual ou superior a 0,87 e EP igual ou inferior a 3,60 (Tabela 3). Existe, portanto, relação não linear entre $\mathrm{C}$ ou L e área foliar, conforme foi observado em folhas de antúrio por Silva et al. (2008). Além disso, a utilização de somente uma dimensão da folha de macieira é estatisticamente aceitável para estimar a área foliar. Situação semelhante foi observada em videira, 
mamoeiro, feijão-de-vagem e girassol (PEDRO JÚNIOR et al., 1986; CAMPOSTRINI; YAMANISHI, 2001; QUEIROGA et al., 2003; MALDANER et al., 2009).

Os modelos linear e quadrático com duas dimensões foliares (comprimento e largura) foram mais eficazes que os com apenas uma das dimensões, sendo classificados entre as quatro primeiras colocações (Tabela 3). Neste caso, nos modelos linear e quadrático, o valor de $\mathrm{R}^{2}$ variou entre 0,99 e 0,98 , EP esteve entre 1,59 e 1,17 e QME variou de 2,54 a 1,37. Além disso, o valor do coeficiente $b$ dos quatro primeiros modelos lineares foi significativo e semelhante, variando de 0,6962 a 0,7029. Nos modelos quadráticos, os coeficientes $b$ e $c$ também foram significativos e semelhantes entre si. Valores de $b$ calculados para modelagem de área foliar de aveleira, pessegueiro e nogueira (CRISTOFORI et al., 2007; DEMIRSOY et al., 2004; TORRI et al., 2009) têm ordem de grandeza semelhante a coeficientes encontrados nos modelos lineares e quadráticos deste trabalho.

Os modelos linear e quadrático com maior significância foram obtidos com dados de folhas de macieiras 'Royal Gala' sob tela antigranizo, gerados pela relação entre AF e C x L (Tabela 3; Figura 1). O modelo linear $\left[\mathrm{AF}=0,6962 * \mathrm{CL}\right.$ ] apresentou $\mathrm{R}^{2}$, EP e QME semelhantes aos do modelo quadrático [AF $\left.=0,7095^{*} \mathrm{CL}-0,0003^{*}(\mathrm{CL})^{2}\right]$, indicando que a área foliar de macieira pode ser estimada com modelos mais simples.

Na Figura 2, os dados estimados pelos modelos selecionados neste trabalho ou pelo modelo proposto por Leonetti et al. (2008) e a área foliar medida via fotografia digital foram comparados com resultados obtidos por meio de planímetro. Verificou-se que os métodos utilizados para estimar a área foliar têm elevada precisão e acurácia, com reduzida variabilidade. Os modelos linear e quadrático selecionados apresentaram coeficientes de regressão significativos, sendo EP e RQME próximos de zero, e os índices de Willmott e de confiança, próximos de 1,0 (Figura 2A e 2B). Além disso, observou-se que a variação da área foliar foi explicada pelo produto C x L em 99\% dos casos. A linha da regressão dos valores medidos versus estimados esteve próxima da linha correspondente à razão $1: 1$, com subestimativa de $3,01 \%$ e $4,84 \%$ para os modelos linear e quadrático, respectivamente. O modelo linear apresentou desempenho igual ao modelo quadrático, indicando que uma equação linear pode ser utilizada para a determinação da área foliar de macieira, como também se verificou em videira (GONÇALVES et al., 2002), mamoeiro (CAMPOSTRINI; YAMANISHI, 2001), fruta-do-conde (LIMA E SILVA et al., 2004) e aveleira (CRISTOFORI et al., 2007).

Nos dados obtidos por fotografia e pelo modelo de Leonetti et al. (2008), os coeficientes da regressão linear indicaram superestimativas de $3,32 \%$ e $0,87 \%$, respectivamente (Figura $2 \mathrm{C}$ e 2D). O emprego de fotografia digital para determinar a área foliar ocorreu em outras culturas, como laranja 'Pera' (GODOY et al., 2007) e soja (ADAMI et al., 2008), em comparação a dados observados com planímetro. Esses autores verificaram que a utilização da imagem digital é aceitável, como método indireto para determinar a área foliar, por apresentar elevada acurácia e precisão, ausência de custo no processamento de imagens, além do baixo custo e da facilidade para a aquisição do equipamento. $\mathrm{O}$ modelo proposto por Leonetti et al. (2008) apresenta características de precisão e desempenho semelhantes aos dois modelos selecionados neste trabalho e ao método de fotografia digital. Sendo assim, métodos diretos (planímetro) ou indiretos (fotografias ou modelos) podem ser utilizados de forma confiável para a obtenção da área foliar de macieira.

TABELA 1- Análise de variância para as variáveis comprimento (C), largura (L), área foliar média (AFm) e índice de forma (IF) de folhas de macieiras 'Royal Gala' e 'Fuji Suprema' sob tela antigranizo e em céu aberto. Vacaria-RS.

\begin{tabular}{lllll}
\hline \multicolumn{1}{c}{ Ambiente } & C & L & AFm $\left(\mathrm{cm}^{2}\right)$ & IF \\
Sob tela antigranizo & $6,1^{\text {ns }}$ & $3,2^{\text {ns }}$ & $17,4^{\text {ns }}$ & $1,89^{\text {ns }}$ \\
Em céu aberto & 6,3 & 3,4 & 19,3 & 1,90 \\
& \multicolumn{3}{c}{ 'Royal Gala' } \\
Sob tela antigranizo & $6,4^{\text {ns }}$ & $4,0^{\text {ns }}$ & $19,9^{\text {ns }}$ & $1,62^{\text {ns }}$ \\
Em céu aberto & 6,1 & 3,7 & 17,3 & 1,68 \\
\hline
\end{tabular}

$\mathrm{ns}$, na linha, indica que não houve diferença significativa entre ambientes, pelo teste $\mathrm{F}(p<0,05)$ 
TABELA 2- Fator de inflação da variância (FIV) e valor de tolerância (T) para detectar a colinearidade entre as variáveis comprimento e largura de folhas de macieiras 'Royal Gala' e 'Fuji Suprema'. Vacaria-RS.

\begin{tabular}{lrc}
\hline \multicolumn{1}{c}{ Cultivar/Ambiente } & FIV & $\mathrm{T}$ \\
& \multicolumn{1}{c}{ Royal Gala' } & \\
Sob tela antigranizo & 8,29 & 0,12 \\
Em céu aberto & 8,51 & 0,12 \\
& 'Fuji Suprema' & \\
Sob tela antigranizo & 12,76 & 0,08 \\
Em céu aberto & 11,69 & 0,09 \\
\hline
\end{tabular}

FIV $>10$ ou $\mathrm{T}<0,10$ indica que a colinearidade influencia os parâmetros de estimativa do modelo.
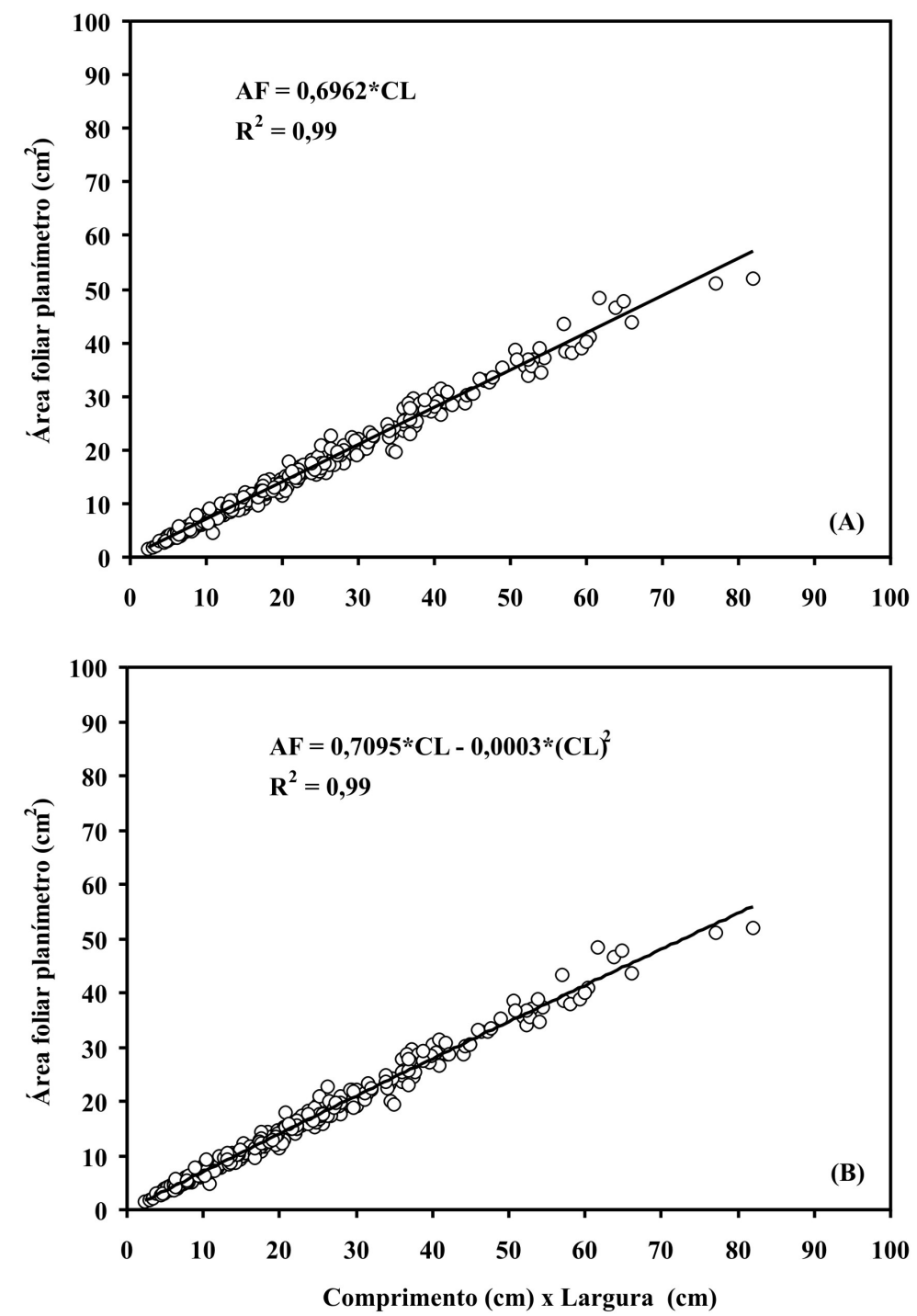

FIGURA 1-Relação linear (A) e quadrática (B) entre área foliar medida em planímetro eletrônico e o produto das dimensões comprimento (C) e largura (L) de folhas de macieiras 'Royal Gala' sob tela antigranizo. Vacaria-RS. 
TABELA 3- Classificação dos modelos linear e quadrático para estimativa de área foliar (AF) de macieiras 'Royal Gala' e 'Fuji Suprema', de acordo com a significância dos coeficientes da regressão, coeficiente de determinação $\left(\mathrm{R}^{2}\right)$, erro-padrão da estimativa $(\mathrm{EP})$ e quadrado médio do erro (QME). Vacaria-RS.

\begin{tabular}{|c|c|c|c|c|c|c|}
\hline Classificação & $\mathrm{Y}=b \mathrm{x}$ & \multicolumn{2}{|c|}{ Coeficiente $b$} & $\mathrm{R}^{2}$ & EP & QME \\
\hline $1^{\circ}$ & $\mathrm{AF}_{\mathrm{GTA}}=b \mathrm{CL}$ & \multicolumn{2}{|c|}{$0,6962 * *$} & 0,99 & 1,18 & 1,38 \\
\hline $2^{o}$ & $\mathrm{AF}_{\mathrm{GCA}}=b \mathrm{CL}$ & \multicolumn{2}{|c|}{$0,6988 * *$} & 0,99 & 1,59 & 2,54 \\
\hline $3^{\circ}$ & $\mathrm{AF}_{\mathrm{GTA}+\mathrm{GCA}}=b \mathrm{CL}$ & \multicolumn{2}{|c|}{$0,6976^{* *}$} & 0,98 & 1,40 & 1,95 \\
\hline $4^{\mathrm{o}}$ & $\mathrm{AF}_{\text {todos }}=b \mathrm{CL}$ & \multicolumn{2}{|c|}{$0,7029 * *$} & 0,98 & 1,45 & 2,09 \\
\hline $5^{\circ}$ & $\mathrm{AF}_{\mathrm{FCA}}=b \mathrm{C}$ & \multicolumn{2}{|c|}{$2,9641 *$} & 0,77 & 4,23 & 17,86 \\
\hline $6^{\circ}$ & $\mathrm{AF}_{\mathrm{GTA}+\mathrm{GCA}}=b \mathrm{C}$ & \multicolumn{2}{|c|}{$2,7742 *$} & 0,77 & 5,11 & 26,10 \\
\hline $7^{\circ}$ & $\mathrm{AF}_{\mathrm{GCA}}=b \mathrm{C}$ & \multicolumn{2}{|c|}{$2,8501 *$} & 0,77 & 5,29 & 27,98 \\
\hline $8^{\circ}$ & $\mathrm{AF}_{\mathrm{GTA}}=b \mathrm{C}$ & \multicolumn{2}{|c|}{$2,6955^{*}$} & 0,76 & 4,88 & 23,87 \\
\hline $9^{\circ}$ & $\mathrm{AF}_{\mathrm{FTA}}=b \mathrm{~L}$ & \multicolumn{2}{|c|}{$5,2063^{*}$} & 0,76 & 5,27 & 27,75 \\
\hline $10^{\circ}$ & $\mathrm{AF}_{\mathrm{FTA}+\mathrm{FCA}}=b \mathrm{~L}$ & \multicolumn{2}{|c|}{$5,0879^{*}$} & 0,75 & 4,95 & 24,55 \\
\hline $11^{\circ}$ & $\mathrm{AF}_{\text {todos }}=b \mathrm{C}$ & \multicolumn{2}{|c|}{$2,8790^{*}$} & 0,75 & 5,21 & 27,15 \\
\hline $12^{\circ}$ & $\mathrm{AF}_{\mathrm{FTA}+\mathrm{FCA}}=b \mathrm{C}$ & \multicolumn{2}{|c|}{$3,1327^{*}$} & 0,74 & 5,08 & 25,83 \\
\hline $13^{\circ}$ & $\mathrm{AF}_{\mathrm{FCA}}=b \mathrm{~L}$ & \multicolumn{2}{|c|}{$4,9427^{*}$} & 0,73 & 4,58 & 20,95 \\
\hline $14^{\circ}$ & $\mathrm{AF}_{\text {todos }}=b \mathrm{~L}$ & \multicolumn{2}{|c|}{$5,1923^{*}$} & 0,73 & 5,44 & 29,6 \\
\hline $15^{\circ}$ & $\mathrm{AF}_{\mathrm{FTA}}=b \mathrm{C}$ & \multicolumn{2}{|c|}{$3,2877^{*}$} & 0,73 & 5,63 & 31,74 \\
\hline $16^{\circ}$ & $\mathrm{AF}_{\mathrm{GCA}}=b \mathrm{~L}$ & \multicolumn{2}{|c|}{$5,3964^{*}$} & 0,73 & 5,70 & 32,45 \\
\hline $17^{\circ}$ & $\mathrm{AF}_{\mathrm{GTA}+\mathrm{GCA}}=b \mathrm{~L}$ & \multicolumn{2}{|c|}{$5,2521 *$} & 0,72 & 5,63 & 31,68 \\
\hline $18^{\circ}$ & $\mathrm{AF}_{\mathrm{GTA}}=b \mathrm{~L}$ & \multicolumn{2}{|c|}{$5,1011 *$} & 0,70 & 5,52 & 30,52 \\
\hline Classificação & $\mathrm{Y}=b \mathrm{x}+c \mathrm{x}^{2}$ & \multicolumn{2}{|c|}{${ }_{b}$ Coeficientes } & $\mathrm{R}^{2}$ & $\mathrm{EP}$ & QME \\
\hline $1^{\circ}$ & $\mathrm{AF}_{\mathrm{GTA}}=b \mathrm{CL}+c \mathrm{CL}^{2}$ & $0,7095 * *$ & $-0,0003 * *$ & 0,99 & 1,17 & 1,37 \\
\hline $2^{\circ}$ & $\mathrm{AF}_{\mathrm{GTA}+\mathrm{GCA}}=b \mathrm{CL}+c \mathrm{CL}^{2}$ & $0,7150 * *$ & $-0,0004 * *$ & 0,98 & 1,38 & 1,91 \\
\hline $3^{\circ}$ & $\mathrm{AF}_{\text {todos }}=b \mathrm{CL}+c \mathrm{CL}^{2}$ & $0,7254 * *$ & $-0,0005 * *$ & 0,98 & 1,42 & 2,03 \\
\hline $4^{\circ}$ & $\mathrm{AF}_{\mathrm{GCA}}=b \mathrm{CL}+c \mathrm{CL}^{2}$ & $0,7205 * *$ & $-0,0005 * *$ & 0,98 & 1,58 & 2,48 \\
\hline $5^{\circ}$ & $\mathrm{AF}_{\mathrm{GTA}}=b \mathrm{C}+c \mathrm{C}^{2}$ & $0,6680 * *$ & $0,2636^{* *}$ & 0,92 & 2,85 & 8,12 \\
\hline $6^{\circ}$ & $\mathrm{AF}_{\mathrm{GTA}+\mathrm{GCA}}=b \mathrm{C}+c \mathrm{C}^{2}$ & $0,6306 * *$ & $0,2738 * *$ & 0,92 & 2,95 & 8,70 \\
\hline $7^{\circ}$ & $\mathrm{AF}_{\mathrm{GCA}}=b \mathrm{CL}+c \mathrm{CL}^{2}$ & $0,6051 * *$ & $0,2820 * *$ & 0,92 & 3,03 & 9,16 \\
\hline $8^{\circ}$ & $\mathrm{AF}_{\mathrm{FTA}}=b \mathrm{~L}+c \mathrm{~L}^{2}$ & $0,9318 * *$ & $0,9072 * *$ & 0,92 & 3,09 & 9,56 \\
\hline $9^{\circ}$ & $\mathrm{AF}_{\mathrm{GCA}}=b \mathrm{~L}+c \mathrm{~L}^{2}$ & $0,6134 * *$ & $1,1648 * *$ & 0,92 & 3,20 & 10,23 \\
\hline $10^{\circ}$ & $\mathrm{AF}_{\mathrm{FTA}+\mathrm{FCA}}=b \mathrm{~L}+c \mathrm{~L}^{2}$ & $1,0334 * *$ & $0,9052 * *$ & 0,91 & 3,02 & 9,14 \\
\hline $11^{\circ}$ & $\mathrm{AF}_{\mathrm{GTA}}=b \mathrm{~L}+c \mathrm{~L}^{2}$ & $0,1747 * *$ & $1,2687 * *$ & 0,91 & 3,03 & 9,17 \\
\hline $12^{\circ}$ & $\mathrm{AF}_{\mathrm{GTA}+\mathrm{GCA}}=b \mathrm{~L}+c \mathrm{~L}^{2}$ & $0,4009 * *$ & $1,2137 * *$ & 0,91 & 3,11 & 9,71 \\
\hline $13^{\circ}$ & $\mathrm{AF}_{\text {todos }}=b \mathrm{~L}+c \mathrm{~L}^{2}$ & $0,8346^{* *}$ & $1,0444 * *$ & 0,90 & 3,36 & 11,29 \\
\hline $14^{\circ}$ & $\mathrm{AF}_{\mathrm{FCA}}=b \mathrm{~L}+c \mathrm{~L}^{2}$ & $0,8952 * *$ & $0,9652 * *$ & 0,89 & 2,91 & 8,45 \\
\hline $15^{\circ}$ & $\mathrm{AF}_{\text {todos }}=b \mathrm{C}+c \mathrm{C}^{2}$ & $0,8215^{* *}$ & $0,2682 * *$ & 0,89 & 3,49 & 12,24 \\
\hline $16^{\circ}$ & $\mathrm{AF}_{\mathrm{FTA}}=b \mathrm{C}+c \mathrm{C}^{2}$ & $0,5203 * *$ & $0,3750 * *$ & 0,89 & 3,60 & 12,99 \\
\hline $17^{\circ}$ & $\mathrm{AF}_{\mathrm{FCA}}=b \mathrm{C}+c \mathrm{C}^{2}$ & $1,2884 * *$ & $0,2330 * *$ & 0,87 & 3,14 & 9,88 \\
\hline $18^{\circ}$ & $\mathrm{AF}_{\mathrm{FTA}+\mathrm{FCA}}=b \mathrm{C}+c \mathrm{C}^{2}$ & $0,9155^{* *}$ & $0,3041^{* *}$ & 0,87 & 3,58 & 12,82 \\
\hline
\end{tabular}

$\mathrm{GTA}=$ 'Royal Gala' sob tela antigranizo; GCA $=$ 'Royal Gala' em céu aberto; FTA $=$ 'Fuji Suprema' sob tela antigranizo; FCA $=$ 'Fuji Suprema' em céu aberto; GTA + GCA = 'Royal Gala'sob tela antigranizo e em céu aberto; FTA + FCA = 'Fuji Suprema' sob tela antigranizo e em céu aberto; todos $=$ dados de GTA, GCA, FTA, FCA juntos. **significativo $(p<0,01)$; *significativo $(p<0,05)$ pelo teste t de Student. 

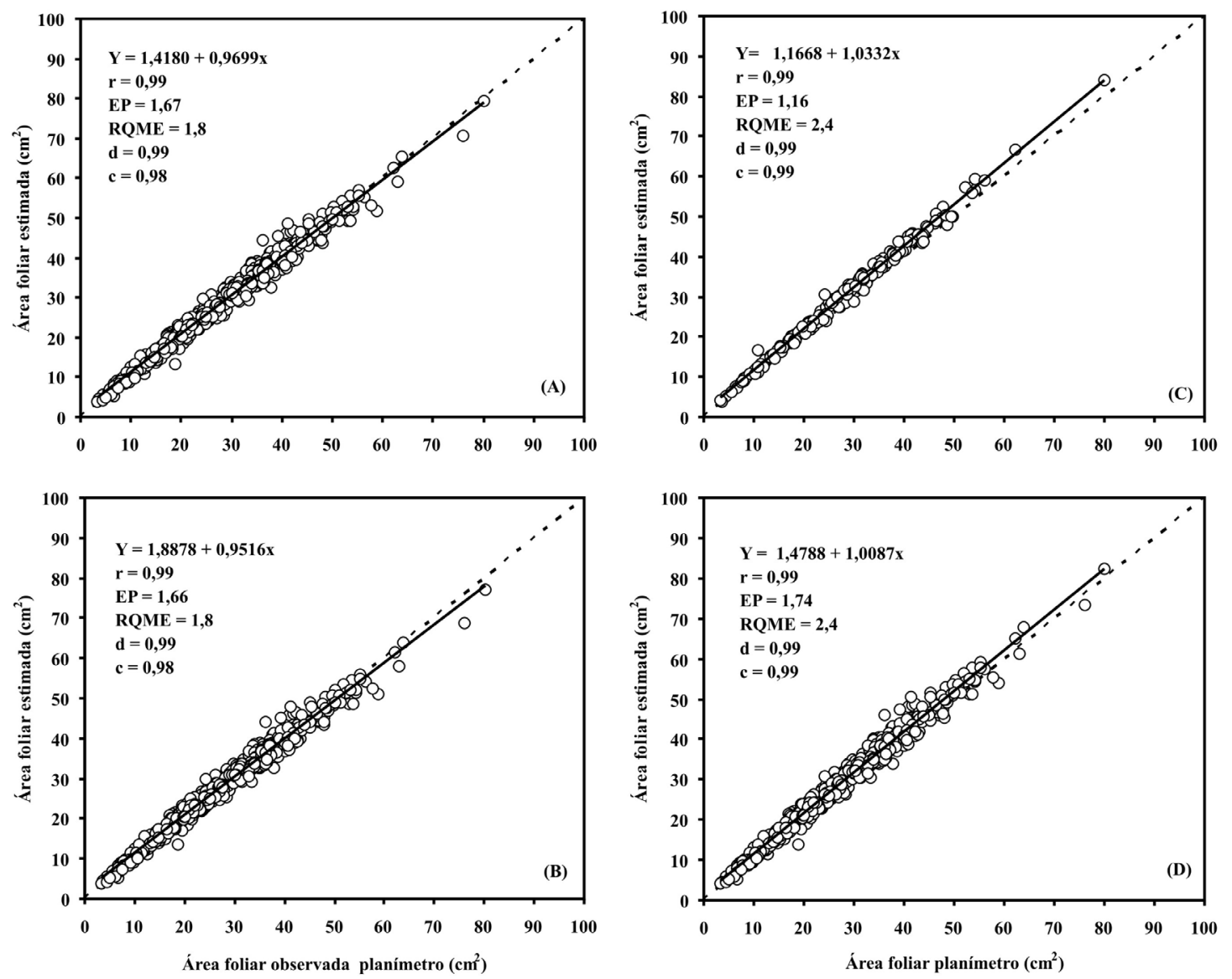

FIGURA 2- Parâmetros de teste para modelos linear (A), quadrático (B), fotografia digital (C) e Leonetti et al. (2008) (D) para determinação da área foliar de macieiras 'Royal Gala' e 'Fuji Suprema'. A linha sólida representa a curva da regressão, e a reta pontilhada representa a relação 1:1 entre valores medidos e estimados. $\mathrm{R}^{2}=$ coeficiente de determinação; $\mathrm{EP}=$ erro padrão da estimativa; $\mathrm{RQME}=$ raiz quadrada do quadrado médio do erro; $\mathrm{d}=$ Índice de Willmott, e c = Índice de confiança. Vacaria-RS,

\section{CONCLUSÕES}

1-Modelos linear e quadrático que consideram as dimensões de comprimento e largura das folhas estimam com acurácia e precisão a área foliar de macieiras 'Royal Gala' e 'Fuji Suprema', tanto sob tela antigranizo quanto em céu aberto.

2-A área foliar de macieira estimada por modelos de regressão que utilizam comprimento e largura de folhas é similar à obtida por planímetro ou fotografia digital.

3-A determinação de área foliar em macieira, por meio de fotografias digitais, fornece dados similares aos obtidos por modelos de estimativa que utilizam dimensões das folhas ou por planímetro.
4-A área foliar de macieira pode ser estimada pelo modelo linear $[\mathrm{AF}=0,6962 * \mathrm{CL}]$ e pelo modelo quadrático $\left[\mathrm{AF}=0,7095 * \mathrm{CL}-0,0003 *(\mathrm{CL})^{2}\right]$.

\section{REFERÊNCIAS}

ADAMI, M.; HASTENREITER, F. A.; FLUMIGNAN, D. L.; FARIA, R. T. Estimativa de área de folíolos de soja usando imagens digitais e dimensões foliares. Bragantia, Campinas, v.67, n.4, p.10531058, 2008.

BALAN, V. Apple trees plantation structure. Notulae Botanicae Horti Agrobotanici Cluj -Napoca, Romania, n.33, p.65-71, 2005. 
BEYHAN, M. A.; UZUN, S.; KANDEMIR, D.; ÖZER, H.; DEMIRSOY, M. A model for predicting leaf area in Young and old leaves of greenhouse type tomato (Lycopersicun esculentum, Mill.) by linear measurements. Journal of Faculty of Agriculture, Kyushu, v.23, n.3, p.154-157, 2008.

BIANCO, S.; PITELli, R. A.; CARVAlHO, L. B. Estimativa da área foliar de Tridax procumbens usando dimensões lineares do limbo foliar. Planta Daninha, Londrina, v.22, n.2, p.247-250, 2004.

CAMARGO, A. P.; SENTELHAS, P. C. Avaliação do desempenho de diferentes métodos de estimativa da evapotranspiração potencial no Estado de São Paulo. Revista Brasileira de Agrometeorologia, São José dos Campos, v.5, n.1, p.89-97, 1997.

CAMPOSTRINI, E.; YAMANISHI, O. K. Estimation of papaya leaf area using the central vein length. Scientia Agricola, Piracicaba, v.58, n.1, p.39-42, 2001.

CARDOSO, L. S. Alterações microclimáticas em vinhedos de Vitis vinífera $\mathrm{L}$. cv. Moscato Giallo pelo uso de cobertura plástica. 2007. 144f. Dissertação (Mestrado em Agrometeorologia) - Faculdade de Agronomia, Universidade Federal do Rio Grande do Sul, Porto Alegre, 2007.

CARDOSO, L. S.; BERGAMASCHI, H.; COMIRAN, F.; CHAVARRIA, G.; MARODIN, G. A. B.; DALMAGO, G. A.; SANTOS, H. P.; MANDELLI, F. Padrões de interceptação de radiação solar em vinhedos com e sem cobertura plástica. Revista Brasileira de Fruticultura, Jaboticabal, v.32, n.1, p.161-171, 2010 .

COELHO FILHO, M. A.; ANGELOCCI, L. R.; VASCONCELOS, M. R. B.; COELHO, E. F. Estimativa da área foliar de plantas de lima ácida 'tahiti' usando métodos não-destrutivos. Revista Brasileira de Fruticultura, Jaboticabal, v.27, n.1, p.163-167, 2005.

COMIRAN, F. Microclima, desenvolvimento e produção de videiras 'Niágara Rosada' em cultivo orgânico sob cobertura plástica. 2009. 74f. (Mestrado em Agrometeorologia) - Faculdade de Agronomia, Universidade Federal do Rio Grande do Sul, Porto Alegre, 2009.
CRISTOFORI, V.; ROUPHAEL, Y.; MENDOZADE GYVES, E.; BIGNAMI, C. A simple model for estimating leaf area of hazelnut from linear measurements. Scientia Horticulturae, Amsterdam, v.113, n.2, p.221-225, 2007.

DE JESUS, W. C.; VALE, F. X. R.; COELHO, R. R.; COSTA, L. C. Comparison of two methods for estimating leaf area index on common bean. Agronomy Journal, Madison, v.93, n.1, p.989-991, 2001.

DEMIRSOY, H. Leaf area estimation in some species of fruit tree by using models as a non-destructive method. Fruits, Cambridge, v.64, n.1, p.45-51, 2009.

DEMIRSOY, H.; DEMIRSOY, L.; UZUN, S.; ERSOY, B. Non-destructive leaf area estimation in peach. European Journal Horticultural Science, Stuttgart, v.69, n.1, p.144-146, 2004.

FAGUNDES, J. L.; DA SILVA, S. C.; PEDREIRA, C. G. S.; CARNEVALLI, R. A.; CARVALHO, C. A. B.; SBRISSIA, A. F.; PINTO, L. F. M. Índice de área foliar, coeficiente de extinção luminosa e acúmulo de forragem em pastagens de Cynodon spp. sob lotação contínua. Pesquisa Agropecuária Brasileira, Brasília, v.36, n.1, p.187-195, 2001.

FLADUNG, M.; RITTER, E. Plant leaf area measurements by personal computers. Journal of Agronomy and Crop Science, Berlin, v.166, n.1, p.69-70, 1991.

FRANCIS, C. A.; RUTGER, J. N.; PALMER, A. F. E. A rapid method for plant leaf area estimation in maize (Zea mays L.). Crop Science, Madison, v.9, n.5, p.537-539, 1969.

GODOY, L. J. G. de; YANAGIWARA, R. S.; BÔAS, R. L. V.; BACKES, C.; LIMA, C. P. de. Análise da imagem digital para estimativa da área foliar em plantas de laranja "Pera". Revista Brasileira de Fruticultura, Jaboticabal, v.29, n.3, 2007.

GONÇALVES, C. A. A.; CHALFUN, N. N. J.; REGINA, M. A.; ALVARENGA, A. A.; SOUZA, M. T.; ABRAHÃO, E. Estimativa de área foliar da videira (Vitis labrusca L. cv. Folha de Figo) sobre diferentes porta-enxertos. Ciência e Agrotecnologia, Lavras, v.26, n.3, p.500-504, 2002. 
JANSSEN, P. H. M.; HEUBERGER, P. S. C. Calibration of process - oriented models. Ecological Modelling, Amsterdam, v.83, n.1-2, p.55-56, 1995.

LAKITAN, B. Empirical model for estimating leaf area in bean (Phaseolus vulgaris L.). Annual Report of the Bean Improvement Cooperative, Fort Collins, v.32, n.1, p.19-21, 1989.

LEONETTI, J. F.; HAWERROTH, F. J.; HERTER, F. G.; PETRI, J. L.; LEITE, G. B.; MARAFON, A. C.; SIMÕES, F. Estimativa da área foliar de macieira (Malus domestica Borkh.) através de dimensões lineares do limbo foliar. In: CONGRESSO BRASILEIRO DE FRUTICULTURA, 20, ANNUAL MEETING OF THE INTERAMERICAN SOCIETY FOR TROPICAL HORTICULTURE, 54., 2008, Vitória-ES. Anais... CD-ROM.

LIMA E SILVA, P. S.; BARBIN, D.; GONÇALVES, R. J. S.; FIRMINO, J. D. C.; FONSECA, I. C. Leaf area estimates of custard apple tree progenies. Revista Brasileira de Fruticultura, Jaboticabal, v.26, n.3, p.558-560, 2004.

LIMA E SILVA, P. S.; GURGEL, F. L.; SILVA, E. S.; DINIZ FILHO, E. T. Equações de regressão para estimação da área foliar de dois clones do cajueiroanão-precoce (Anacardium occidentale L.). Ciência Agronômica, Fortaleza, v.31, n.12, p.89-95, 2000.

LU, H. Y.; LU, C. T.; WEI, M. L.; CHAN, F. L. Comparison of different models for nondestructive leaf area estimation in taro. Agronomy Journal, Amsterdam, v.96, p.448-453, 2004.

MALDANER, I. C.; HELDWEIN, A. B.; LOOSE, L. H.; LUCAS, D. D. P.; GUSE, F. I.; BORTOLUZZI, M. P. Modelos de determinação não destrutiva da área foliar em girassol. Ciência Rural, Santa Maria, v.39, n.5, p.1356-1361, 2009.

MIELKE, M. S.; HOFFMAN, A.; ENDRES, J. C.; FACHINELLO, J. C. Comparação de métodos de laboratório e de campo para estimação da área foliar em fruteiras silvestres. Scientia Agricola, Piracicaba, v.52, n.1, p. 82-88, 1995.

MIDDLETON, S.; McWATERS, A. Hail netting of apple orchards. Australian Experience. Compact Fruit Tree, Middleburg, v.35, n.2, p.51-55, 2002.
O’NEAL, M. E.; LANDIS, D. A.; ISAACS, R. An inexpensive, accurate method for measuring leaf area and defoliation through digital image analysis. Journal of Economic Entomology, Lanham, v.95, n.6, p.1190-1194, 2002.

PALMER, J. W. The measurements leaf area on apple tree. The Journal of Horticultural Science, London, v.62, n.1, p.5-10, 1987.

PEDRO JÚNIOR, M. J.; RIBEIRO, I. J. A.; MARTINS, F. P. Determinação da área foliar em videira cultivar Niagara Rosada. Bragantia, Campinas, v.45, n.1, p. 199-204, 1986.

PEKSEN, E. Non-destructive leaf area estimation model for faba bean (Vicia faba L.) Scientia Horticulturae, Amsterdam, v.113, n.4, p.322-328, 2007.

PINTO JÚNIOR, O. B; SANCHES, L.; LOBO, F. A.; BRANDÃO, A. A.; NOGUEIRA, J. S. Leaf area index of a tropical semi-deciduous forest of the southern Amazon Basin. International Journal of Biometeorology, Lisse,v.55, n.2, p.109-118, 2010.

PINTO, A. C. R.; RODRIGUES, T. J. D.; BARBOSA, J. C.; LEITE, I. C. Leaf area prediction models for Zinnia elegans Jacq., Zinnia haageana Regel and 'Profusion Cherry'. Scientia Agricola, Piracicaba, v.61, n.1, p.47-52, 2004.

POTDAR, M. Y.; PAWAR, K. R. Non-destructive leaf area estimation in banana. Scientia Horticulturae, Amsterdam, v.45, n.3-4, p.251-254, 1991.

QUEIROGA, J. L.; ROMANO, E. D. U.; SOUZA, J. R. P.; MIGLIORANZA, E. Estimativa da área foliar do feijão-de-vagem (Phaseolus vulgaris L.) por meio da largura máxima do folíolo central. Horticultura Brasileira, Campinas, v.21, n.1, p.64-68, 2003.

SCHNEIDER, P. R. Análise de regressão aplicada à Engenharia Florestal. 2.ed. Santa Maria: Centro de Pesquisas Florestais, UFSM, 1998. 236p.

SERDAR, Ü.; DEMIRSOY, H. Non-destructive leaf area estimation in chestnut. Scientia Horticulturae, Amsterdam, v.108, n.2, p.227-230, 2006.

SILVA, S. H. M. G.; LIMA, J. D.; BENDINI, H. N.; NOMURA, E. S.; MORAES, W. S. Estimativa da área foliar do antúrio com o uso de funções de regressão. Ciência Rural, Santa Maria, v.38, n.1, p.243-246, 2008. 
TAVARES-JÚNIOR, J. E.; FAVARIN, J. L.; DOURADO-NETO, D.; MAIA, A. H. N.; FAZUOLI, L. C.; BERNARDES, M. S. Análise comparativa de métodos de estimativa de área foliar em cafeeiro. Bragantia, Campinas, v.61, n.2, p.199-203, 2002.

TORRI, S. R.; DESCALZI, C.; FRUSSO, E. Estimation of leaf area in pecan cultivars (Carya illinoinensis). Ciencia e Investigación Agraria, Santiago, v.36, n.1, p.53-58, 2009.

UZUN, S.; ÇELIK, H. Leaf area prediction models (Uzçelik-I) for different horticultural plants. Turkish Journal of Agriculture and forestry, Turquia, v.23, p.645-650, 1999.
VALE, F. X. R.; FERNANDES FILHO, E. I.; LIBERATO, J. R. QUANT: a software plant disease severity assessment. In: INTERNATIONAL CONGRESS OF PLANT PATHOLOGY, 8., 2003, Christchurch. Proceedings... p.105

WIERSMA, J. V.; BAILEY, T. B. Estimation of leaflet, trifoliate, and total leaf areas of soybeans. Agronomy Journal, Madison, v.67, n.1, p.26-30, 1975.

WILLMOTT, C. J. On the validation of models. Physical Geography, Columbia, v.2, n.2, p.184194, 1981. 